\title{
Whole Brain Voxel-Wise Analysis of Cerebral Retention of Beta-Amyloid in Cognitively Normal Older Adults Using ${ }^{18}$ F-Florbetaben
}

\author{
Yoo Hyun Um ${ }^{1}$, Woo Hee Choi ${ }^{2}$, Won Sang Jung ${ }^{3}$, Young Ha Park ${ }^{3}$, Chang-Uk Lee ${ }^{4}$, and Hyun Kook Lim ${ }^{5}$ \\ 1'Department of Psychiatry, St. Vincent's Hospital, College of Medicine, The Catholic University of Korea, Seoul, Republic of Korea \\ ${ }^{2}$ Department of Radiology, Division of Nuclear Medicine, St. Vincent's Hospital, College of Medicine, The Catholic University of Korea, Seoul, \\ Republic of Korea \\ ${ }^{3}$ Department of Radiology, St. Vincent's Hospital, College of Medicine, The Catholic University of Korea, Seoul, Republic of Korea \\ ${ }^{4}$ Department of Psychiatry, Seoul St. Mary's Hospital, College of Medicine, The Catholic University of Korea, Seoul, Republic of Korea \\ ${ }^{5}$ Department of Psychiatry, Yeouido St. Mary's Hospital, College of Medicine, The Catholic University of Korea, Seoul, Republic of Korea
}

Objective Recently developed 18F-labelled amyloid beta (A $\beta$ ) positron emission tomography (PET) tracers have demonstrated potentials to enable more prevalent application of amyloid imaging in the clinical setting. The aim of this study is to demonstrate cerebral retention of $\mathrm{A} \beta$ in cognitively normal older adults, by implementing voxel-based analysis on images acquired from ${ }^{18} \mathrm{~F}$-Florbetaben amyloid PET.

Methods Fifty cognitive normal elderly subjects were recruited and included in the study. Demographic data and cognitive measurements were collected. Magnetic resonance imaging (MRI) and ${ }^{18} \mathrm{~F}$-Florbetaben PET data were obtained followed by whole brain voxelbased analysis.

Results Compared to the florbetaben (FBB) (-) counterpart, FBB (+) showed significantly higher A $\beta$ deposition in the brain regions comprising anterior cingulate, middle cingulate, posterior cingulate and precuneus (family wise error corrected $\mathrm{p}<0.05$ ). There was no significant correlation between amyloid retention and cognitive functions.

Conclusion Our results confirms previous results regarding $\mathrm{A} \beta$ deposition by using ${ }^{18} \mathrm{~F}$-Florbetaben, demonstrating potentials in application of ${ }^{18} \mathrm{~F}$-Florbetaben PET imaging in clinical settings. Psychiatry Investig 2017;14(6):883-886

Key Words Alzheimer's disease, Amyloid-beta protein, Florbetaben, Cognition.

\section{INTRODUCTION}

Alzheimer disease $(\mathrm{AD})$ is a neurodegenerative disease notorious for its chronic and debilitating course, and it is strongly correlated with cognitive impairments and behavioral disturbances. Among the several pathophysiological causes of $\mathrm{AD}$, cerebral retention of toxic amyloid- $\beta(\mathrm{A} \beta)$ peptides in soluble or intraneuronal forms has been repeatedly suggested, with amyloid plaques known to provoke synaptic injury and neurodegeneration associated with $\mathrm{AD}{ }^{1}$

Received: August 14, 2016 Revised: October 10, 2016

Accepted: October 27, 2016 Available online: June 16, 2017

$\triangle$ Correspondence: Hyun Kook Lim, MD, PhD

Department of Psychiatry, Yeouido St. Mary's Hospital, College of Medicine, The Catholic University of Korea, 10 63-ro, Yeongdeungpo-gu, Seoul 07345, Republic of Korea

Tel: +82-31-249-7150, Fax: +82-2-780-6577, E-mail: drblues@catholic.ac.kr

(a) This is an Open Access article distributed under the terms of the Creative Commons Attribution Non-Commercial License (http://creativecommons.org/licenses/by$\mathrm{nc} / 4.0$ ) which permits unrestricted non-commercial use, distribution, and reproduction in any medium, provided the original work is properly cited.
Introduction of amyloid positron emission tomography (PET) has contributed to the unraveling of the underlying pathological mechanisms of $\mathrm{AD}^{2}$ To date, the most widely used and studies A $\beta$ PET tracer has been, 11C-labelled Pittsburgh compound-B (PiB). ${ }^{3}$ However, considering short half-life of carbon radioisotope (20 min), use of $\mathrm{PiB}$ is largely restricted, hindering it from active application in clinical settings. ${ }^{4}$ In these regards, 18F-labelled A $\beta$ PET tracers longer half-life of $110 \mathrm{~min}$ (florbetaben, florbetapir, flutemetamol), have been discovered and gained US Food and Drug Administration approval for use in clinical practice. ${ }^{4}$ Among the $3 \mathrm{~F}$ labeled $\mathrm{A} \beta$ tracers, florbetaben (FBB) was the first $18 \mathrm{~F}$-labeled $\mathrm{A} \beta$ tracer studied in humans. ${ }^{4}$ Several prior works showed high sensitivity and specificity (90\% sensitivity and $80 \%$ specificity) in discriminating $\mathrm{AD}$ from the normal controls. ${ }^{5}$ However, as compared to the studies using PiB, there is no comparison study on cerebral retention pattern between the cognitively intact elderly according to their $A \beta$ retention status. As $A \beta$ protein is known to accu- 
mulate for 10-15 years before the emergence of cognitive impairments in $\mathrm{AD}$, measuring $\mathrm{A} \beta$ retention pattern in the cognitively normal older adults would help to understand more sophisticated pathophysiological process underpinning $\mathrm{AD}$ trajectory. ${ }^{6}$ In this study we used whole brain voxel wise analysis to measure $A \beta$ retention pattern in cognitively normal older adults. As compared to region of interest (ROI), the benefits of the voxel wise analysis is to measure whole brain $A \beta$ without a priori consumption. We hypothesized that the retention patterns will differ between FBB (+) and (-) group in the posterior cingulate $(\mathrm{PCC})$, precunes $(\mathrm{PRC})$, anterior cingulate (ACC), lateral temporal (LT) and frontal cortex (FRC) as in the previous voxel based study using the PiB.?

\section{METHODS}

\section{Subjects}

In this study, 50 elderly participants with intact cognition were recruited from the community advertisements and from the Catholic Geriatric Neuroimaging Database. The inclusion criteria of the subjects were: 1 ) subjects aged $>60$ years, 2) and Clinical Dementia Rating $=0,{ }^{8}$ and 3) Mini-Mental Status Examination score $>27$. Participants with any of the neurological, medical, or psychiatric diseases were excluded from the study.

The cognitive functions were tested using the Korean version of the Consortium to Establish a Registry for Alzheimer's Disease (CERAD-K). ${ }^{9}$

\section{PET acquisition}

Combined PET/CT in-line systems (Gemini TF, Philips
Healthcare, Best, The Netherlands) were used to acquire all data. An average of $300 \mathrm{MBq}$ FBB was injected intravenously, and the scan was initiated 90 minutes later. A low dose CT scan was performed for attenuation correction and was immediately followed by PET imaging in three-dimensional mode for 20 minutes. Fixation of the subject's head was done with a head holder to minimize motion artifacts. The standard ordered subset expectation maximization (OSEM) algorithm (subset 33, integration 3) was utilized for the reconstruction of PET images. To determine amyloid positivity, whole-brain visual assessment used methodology adapted from previously described techniques. ${ }^{5}$

\section{MRI acquisition}

MRI images were obtained at the MRI center of the St. Vincent Hospital using a 3T Siemens Verio machine, and 8-channel Siemens head coil. A standard high-resolution T1-weighted volumetric magnetization prepared rapid gradient echo scans (MP-RAGE) sequence was acquired in axial orientation (160 slices, $256 \times 240,1 \mathrm{~mm}$ isotropic).

\section{Image preprocessing and statistical analysis}

Reorientation of high-resolution, skull-cropped, MP-RAGE images was conducted with the AC-PC line and the medial longitudinal fissure as points of alignment. The ICBM 152 template (Montreal Neurological Institute) ${ }^{10}$ of the SPM8 software package (http://www.fil.ion.ucl.ac.uk/spm/software/spm8/) was utilized for the normalization of the acquired images. A general linear model (GLM) analysis was used for the group comparison between the FBB (+) and the FBB (-) groups while controlling for age, gender and education. The threshold was

Table 1. Demographic and clinical characteristics of study participants

\begin{tabular}{lccc}
\hline & FBB (-) group $(\mathrm{N}=34)$ & FBB (+) group $(\mathrm{N}=16)$ & p-value \\
\hline Age (years \pm SD) & $67.2 \pm 4.3$ & $68.2 \pm 5.4$ & 0.84 \\
Education (years \pm SD) & $9.6 \pm 2.1$ & $9.5 \pm 2.4$ & 0.64 \\
Sex (M : F) & $13: 21$ & $5: 11$ & 0.41 \\
CDR (SD) & 0 & 0 & 0.87 \\
CERAD-K Battery (SD) & & & 0.73 \\
VF & $14.3 \pm 2.7$ & $13.7 \pm 3.2$ & 0.81 \\
BNT & $11.1 \pm 3.1$ & $12.1 \pm 2.3$ & 0.83 \\
MMSE & $28.7 \pm 1.2$ & $28.2 \pm 1.1$ & 0.79 \\
WLM & $17.4 \pm 3.2$ & $17.1 \pm 3.4$ & 0.13 \\
CP & $8.3 \pm 2.5$ & $8.2 \pm 1.9$ & 0.9 \\
WLR & $7.2 \pm 1.6$ & $6.9 \pm 1.8$ & 0.21 \\
WLRc & $11.1 \pm 1.4$ & $11.5 \pm 1.7$ & $7.2 \pm 2.9$ \\
CR & $6.9 \pm 2.8$ & & 0 \\
\hline
\end{tabular}

FBB: Florbetaben, SD: standard deviation, CDR: Clinical Dementia Rating, CERAD-K: the Korean version of Consortium to Establish a Registry for Alzheimer's Disease, VF: verbal fluency, BNT: 15-item Boston Naming Test, MMSE: Mini Mental Status Examination, WLM: word list memory, CP: Constructional Praxis, WLR: word list recall, WLRc: word list recognition, CR: constructional recall 
set at $\mathrm{p}<0.05$ [family wise error (FWE)] to overcome errors pertaining to multiple comparisons.

\section{RESULTS}

Table 1 showed the demographic data of the study participants. There was no significant differences between the FBB $(+)$ and the FBB (-) group in demographic and the cognitive and behavioral measurements. In the group analysis, The FBB (+) group showed significantly higher amyloid retention pattern in the ACC, PRC, middle cingulate and PCC (Figure 1, Supplementary Table 1 in the online-only Data Supplement, FWE corrected $\mathrm{p}<0.05)$. However, these results were expanded to ACC, PRC, PCC FRC, and LT compared with the FBB (-) group when the level of significant adjusted to false discovery rate $\mathrm{p}<0.05$ (Supplementary Figure 1 online-only Data Supplement). There was no significant correlation between the cognitive function scores measured by CERAD-K and the amyloid retention in the FBB (+) group. The detailed coordinates of group differences showed in the Supplemental Table 1 (online-only Data Supplement).

\section{DISCUSSION}

To our knowledge, this the first article to report results from whole brain voxel-based analysis of $\mathrm{A} \beta$ deposition utilizing ${ }^{18} \mathrm{~F}$-Florbetaben on cognitively normal elderly subjects. According to our results, several notable brain regions in FBB $(+)$ group demonstrated significantly higher $A \beta$ retention pattern when compared with that of FBB (-) group.
Due to a short history of $18 \mathrm{~F}-$ labeled ligands in amyloid imaging research, there have been relatively few articles on voxel-based analysis of cerebral $\mathrm{A} \beta$ deposition using $18 \mathrm{~F}$-labeled ligands. To list a few, a recent article utilizing ${ }^{18} \mathrm{~F}$-Florbetapir discussed the potential role interaction between $A \beta$ deposition and phosphorylated tau played on the decline of metabolism certain brain regions. ${ }^{11}$ Such interaction was most evident in the temporal cortex, frontal cortex and both cingulate cortices. ${ }^{11}$ Our results are in line with the aforementioned results, where amyloid retention was most evident the both ACC, PRC, PCC, FRC and LT. Studies on major depressive disorder patients with mild cognitive impairment (MCI) and Down syndrome and the pattern of $A \beta$ retention in these two cohorts explored with ${ }^{18} \mathrm{~F}$-Florbetapir yielded some meaningful results that accentuated the diagnostic utility of amyloid imaging in the prevention and early intervention of dementia. ${ }^{12,13}$ As with ${ }^{18} \mathrm{~F}$-Flutemetamol, a recent research utilized the ligand in discriminating MCI subjects into amyloid positive and negative groups, exploring possible differential factor between two groups including cortical metabolism and hippocampal volume by adopting voxel-wise surface maps. ${ }^{14}$

The brain regions with prominent $\mathrm{A} \beta$ deposition in $\mathrm{FBB}(+)$ group were regions that have been known to comprise the default mode network (DMN) ${ }^{15}$ which is frequently suggested to be areas of great connectivity where incipient $A \beta$ deposition takes place. ${ }^{16}$ Despite the aforementioned $A \beta$ burden in the $\mathrm{DMN}$, which plays an integral role in the self-referential processing, attention and working memory, ${ }^{17}$ there was no significant correlation between the cognitive function scores and $A \beta$ retention in our study, deviant from our original hypoth-

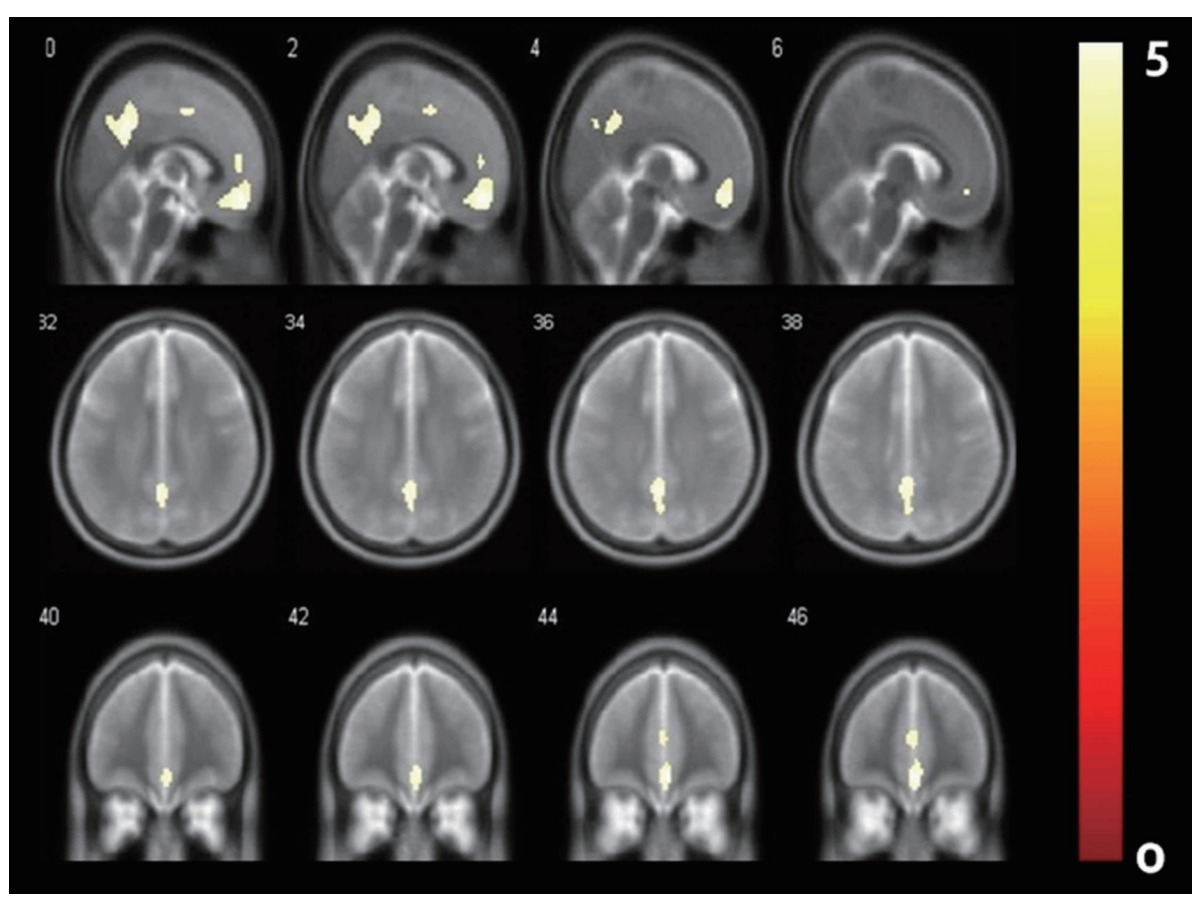

Figure 1. Results from voxel-wise analysis comparing amyloid deposition pattern between FBB (+) and FBB (-) group by using ${ }^{18} \mathrm{~F}$-Florbetben brain PET. The results were color-coded by FWE-corrected T-statistic values (FWE corrected $p<0.05)$. FBB: Florbetaben, FWE: Family wise error. 
esis. This phenomenon could be explained by relatively preserved brain reserve and cognitive function in this group. ${ }^{6,18}$ Moreover, this group of participants could be subjects with high basal cerebral metabolism that has strength to withstand the adverse effects of $A \beta$ retention. ${ }^{18}$

Our study has several limitations that must be taken into consideration. Our sample sizes were small, making our results difficult to generalize. Moreover, extension of the study to include data on apolipoprotein E genotyping and each regional standard uptake value ratio could have added more strength in our results. Further studies including the aforementioned variables could increase validity and objectivity. Lastly, although validated to a certain level proving accuracy in detecting a $\mathrm{A} \beta$ deposition, ${ }^{19}$ additional standardized data on ${ }^{18} \mathrm{~F}$-Florbetaben could be of help for more active utilization of this ligand in the clinical setting.

In summary, our study results are in line with previous findings where $\mathrm{A} \beta$ deposition mainly affected brain regions with active connectivity at rest. Moreover, further exploration on the relationship between brain reserve and amyloid burden will unravel underlying pathologic mechanism that precedes actual manifestation of $\mathrm{AD}$ symptoms.

\section{Supplementary Materials}

The online-only Data Supplement is available with this article at https://doi.org/10.4306/pi.2017.14.6.883.

\section{Acknowledgments}

This research was supported by Basic Science Research Program through the National Research Foundation of Korea (NRF) funded by the Ministry of Science, ICT \& Future Planning (NRF-2015R1C1A1A02036578).

This research was supported by a grant of the Korea Health Technology R\&D Project through the Korea Health Industry Development Institute (KHIDI), funded by the Ministry of Health \& Welfare, Republic of Korea (grant number: $\mathrm{HC15C1405).}$

\section{REFERENCES}

1. Hardy J, Selkoe DJ. The amyloid hypothesis of Alzheimer's disease: progress and problems on the road to therapeutics. Science 2002;297: 353-356.

2. Schmidt ME, Chiao P, Klein G, Matthews D, Thurfell L, Cole PE, et al. The influence of biological and technical factors on quantitative analysis of amyloid PET: points to consider and recommendations for controlling variability in longitudinal data. Alzheimers Dement 2015;11: 1050-1068.
3. Klunk WE, Engler H, Nordberg A, Wang Y, Blomqvist G, Holt DP, et al. Imaging brain amyloid in Alzheimer's disease with Pittsburgh Compound-B. Ann Neurol 2004;55:306-319.

4. Syed YY, Deeks E. [(18)F]Florbetaben: a review in beta-amyloid PET imaging in cognitive impairment. CNS Drugs 2015;29:605-613.

5. Sabri O, Sabbagh MN, Seibyl J, Barthel H, Akatsu H, Ouchi Y, et al. Florbetaben PET imaging to detect amyloid beta plaques in Alzheimer's disease: phase 3 study. Alzheimers Dement 2015;11:964-974.

6. Lim HK, Nebes R, Snitz B, Cohen A, Mathis C, Price J, et al. Regional amyloid burden and intrinsic connectivity networks in cognitively normal elderly subjects. Brain 2014;137:3327-3338.

7. Aizenstein HJ, Nebes RD, Saxton JA, Price JC, Mathis CA, Tsopelas ND, et al. Frequent amyloid deposition without significant cognitive impairment among the elderly. Arch Neurol 2008;65:1509-1517.

8. Morris JC. The Clinical Dementia Rating (CDR): current version and scoring rules. Neurology 1993;43:2412-2414.

9. Lee JH, Lee KU, Lee DY, Kim KW, Jhoo JH, Kim JH, et al. Development of the Korean version of the Consortium to Establish a Registry for Alzheimer's Disease Assessment Packet (CERAD-K): clinical and neuropsychological assessment batteries. J Gerontol B Psychol Sci Soc Sci 2002;57:P47-P53.

10. Ashburner J, Friston KJ. Unified segmentation. Neuroimage 2005;26: 839-851.

11. Pascoal TA, Mathotaarachchi S, Mohades S, Benedet AL, Chung CO, Shin M, et al. Amyloid-[beta] and hyperphosphorylated tau synergy drives metabolic decline in preclinical Alzheimer's disease. Mol Psychiatry 2017;22:306-311.

12. Wu KY, Liu CY, Chen CS, Chen CH, Hsiao IT, Hsieh CJ, et al. Beta-amyloid deposition and cognitive function in patients with major depressive disorder with different subtypes of mild cognitive impairment: 18(F)florbetapir (AV-45/Amyvid) PET study. Eur J Nucl Med Mol Imaging 2016;43:1067-1076.

13. Sabbagh MN, Chen K, Rogers J, Fleisher AS, Liebsack C, Bandy D, et al. Florbetapir PET, FDG PET, and MRI in Down syndrome individuals with and without Alzheimer's dementia. Alzheimers Dement 2015;11: 994-1004.

14. Hanseeuw B, Dricot L, Lhommel R, Quenon L, Ivanoiu A. Patients with amyloid-negative mild cognitive impairment have cortical hypometabolism but the hippocampus is preserved. J Alzheimers Dis 2016;53: 651-660.

15. Greicius M. Resting-state functional connectivity in neuropsychiatric disorders. Curr Opin Neurol 2008;21:424-430.

16. Myers N, Pasquini L, Göttler J, Grimmer T, Koch K, Ortner M, et al. Within-patient correspondence of amyloid- $\beta$ and intrinsic network connectivity in Alzheimer's disease. Brain 2014;137:2052-2064.

17. Whitfield-Gabrieli S, Ford JM. Default mode network activity and connectivity in psychopathology. Annu Rev Clin Psychol 2012;8:49-76.

18. Cohen AD, Price JC, Weissfeld La, James J, Rosario BL, Bi W, et al. Basal cerebral metabolism may modulate the cognitive effects of Abeta in mild cognitive impairment: an example of brain reserve. J Neurosci 2009; 29:14770-14778.

19. Syed YY, Deeks E. [(18)F]Florbetaben: a review in $\beta$-Amyloid PET imaging in cognitive impairment. CNS Drugs 2015;29:605-613. 